\title{
The Transform between the space of observed values and the space of possible values of the parameter
}

\author{
Sergey I. Bityukov*i \\ Institute for high energy physics, 142281 Protvino, Russia \\ Email: Serguei.Bitioukovecern.ch \\ Nikolai V. Krasnikov \\ Institute for nuclear research RAS, Moscow, Russia \\ E-mail: Nikolai.Krasnikov@cern.ch \\ Vera V. Smirnova \\ Institute for high energy physics, 142281 Protvino, Russia E-mail: \\ Vera.Smirnovadihep.ru
}

Vera A. Taperechkina

Moscow State University of instrument engineering and computer science, Moscow, Russia

In ref [1] the notion of statistically dual distributions is introduced. The reconstruction of confidence density [2] for the location parameter for several pairs of statistically dual distributions (Poisson and Gamma, normal and normal, Cauchy and Cauchy, Laplace and Laplace) in the case of single observation of the random variable is a unique. It allows to introduce the Transform between the space of observed values and the space of possible values of the parameter.

XI International Workshop on Advanced Computing and Analysis Techniques in Physics Research April 23-27 2007

Amsterdam, the Netherlands

\footnotetext{
*Speaker.

${ }^{\dagger}$ This work has been supported by grants RFBR 07-02-00256-a.
} 


\section{Introduction}

As it is shown in refs. [3, 牙, in the framework of frequentist approach we can construct the probability distribution of the possible magnitudes of the Poisson distribution parameter to give the observed number of events $\hat{n}$ in a Poisson stream of events. This distribution, which can be called a confidence density function of a parameter, is described by a Gamma-distribution with the probability density function which looks like Poisson distribution of probabilities. This is the reason for naming this pair of distributions as statistically dual distributions. Also, the interrelation between the Poisson and Gamma distributions allows to reconstruct the confidence density of the Poisson distribution parameter in a unique way [1] and, correspondingly, to construct any confidence interval for the parameter ${ }^{1}$.

According to B. Efron [5] the confidence density is the fiducial [6] distribution of the parameter. This distribution is considered as a genuine a posterior density for the parameter without prior assumptions. More detail the confidence distributions is considered in ref. [7].

The same relation, which allows one to reconstruct the confidence density of a parameter in a unique way, exists between several pairs of statistically self-dual distributions (normal and normal, Laplace and Laplace and Cauchy and Cauchy). As consequence, the Transform between the space of realizations of the random variable and the space of possible values of the parameter takes place [2, 8] in this case.

Note that the posterior distribution of the parameter is also used for the definition of conjugate families in the Bayesian approach. The interrelation between the statistically dual distributions and conjugate families is discussed in ref. [2].

The notion "statistically dual distributions" is introduced in the next Section. Section 3 describes the Transform between the space of realizations of the random variable and the space of possible values of the parameter. The method of the confidence density construction for signal with known background is shown in Section 4 as an example of the Transform application. In Section 5 the confidence density and the Bayes' Theorem are used for estimation of the uncertainty in distinguishing of two simple hypotheses under the experiment planning [Ð].

\section{Statistically dual distributions}

Let us define statistically dual distributions.

Definition 1: Let $\phi(x, \theta)$ be a function of two variables. If the same function can be considered both as a family of the probability density functions (pdf) $f(x \mid \theta)$ of the random variable $x$ with parameter $\theta$ and as another family of pdf's $\tilde{f}(\theta \mid x)$ of the random variable $\theta$ with parameter $x$ (i.e. $\phi(x, \theta)=f(x \mid \theta)=\tilde{f}(\theta \mid x))$, then this pair of families of distributions can be named as statistically dual distributions.

The statistical duality of Poisson and Gamma-distributions follows from simple discourse.

Let us consider the Gamma-distribution $\Gamma_{1, n+1}$ with probability density [9]

\footnotetext{
${ }^{1}$ If we have the procedure which states the one-to-one conformity between the observed value of random variable and the confidence interval of any level of significance then we can reconstruct the confidence density of the parameter in single way. Confidence density contains more information than confidence interval.
} 


$$
g_{n}(\mu)=\frac{\mu^{n}}{n !} e^{-\mu}, \mu>0, n>-1 .
$$

It is a common supposition that the probability of observing $n$ events in the experiment is described by Poisson distribution with parameter $\mu$, i.e.

$$
f(n \mid \mu)=\frac{\mu^{n}}{n !} e^{-\mu}, \mu>0, n \geq 0 .
$$

One can see that if the parameter and variable in Eq. (2.1) and Eq. (2.2) are exchanged, in other respects the formulae are identical. As a result these distributions (Gamma and Poisson) are statistically dual distributions.

These distributions are connected by the identity [3] (see, also, this identity in another form in refs. [10, 11, 12])

$$
\sum_{i=\hat{n}+1}^{\infty} f\left(i \mid \mu_{1}\right)+\int_{\mu_{1}}^{\mu_{2}} g_{\hat{n}}(\mu) d \mu+\sum_{i=0}^{\hat{n}} f\left(i \mid \mu_{2}\right)=1
$$

i.e.

$$
\sum_{i=\hat{n}+1}^{\infty} \frac{\mu_{1}^{i} e^{-\mu_{1}}}{i !}+\int_{\mu_{1}}^{\mu_{2}} \frac{\mu^{\hat{n}} e^{-\mu}}{\hat{n} !} d \mu+\sum_{i=0}^{\hat{n}} \frac{\mu_{2}^{i} e^{-\mu_{2}}}{i !}=1
$$

for any real $\mu_{1} \geq 0$ and $\mu_{2} \geq 0$ and non-negative integer $\hat{n}$. We can suppose that $\hat{n}$ is a number of observed events.

The definition of the confidence interval $\left(\mu_{1}, \mu_{2}\right)$ for the Poisson distribution parameter $\mu$ using [3, 2]

$$
P\left(\mu_{1} \leq \mu \leq \mu_{2} \mid \hat{n}\right)=P\left(i \leq \hat{n} \mid \mu_{1}\right)-P\left(i \leq \hat{n} \mid \mu_{2}\right),
$$

where $P(i \leq \hat{n} \mid \mu)=\sum_{i=0}^{\hat{n}} \frac{\mu^{i} e^{-\mu}}{i !}$, allows one to show that a Gamma-distribution $\Gamma_{1,1+\hat{n}}$ is the probability distribution of different values of $\mu$ parameter of Poisson distribution under condition that the observed value of the number of events is equal to $\hat{n}$, i.e. $\Gamma_{1,1+\hat{n}}$ is the confidence density of the parameter $\mu$. This definition is consistent with the identity Eq. (2.3). Note, if we suppose in Eq. (2.3) that $\mu_{1}=\mu_{2}$ we have a conservation of probability. The right-hand side of Eq. (2.4) determines the frequentist sense of this definition.

Let us consider, for example, the Cauchy distribution with unknown parameter $\theta$ and known parameter $b$. Here we also can exchange the parameter $\theta$ and variable $x$ while conserving the same formula of the probability distribution.

The probability density of the Cauchy distribution is

$$
C(x \mid \theta)=\frac{b}{\pi\left(b^{2}+(x-\theta)^{2}\right)} .
$$

The probability density of its statistically dual distribution is also the Cauchy distribution:

$$
\tilde{C}(\theta \mid x)=\frac{b}{\pi\left(b^{2}+(x-\theta)^{2}\right)} .
$$


In this a way the Cauchy distribution can be named as statistically self-dual distribution. An identity like Eq. (2.3) also holds in another form,

$$
\int_{-\infty}^{\hat{x}-\delta_{1}} C(x \mid \hat{x}) d x+\int_{\hat{x}-\delta_{1}}^{\hat{x}+\delta_{2}} \tilde{C}(\theta \mid \hat{x}) d \theta+\int_{\hat{x}+\delta_{2}}^{\infty} C(x \mid \hat{x}) d x=1,
$$

where $\hat{x}$ is the observed value of random variable $x, \hat{x}-\delta_{1}$ and $\hat{x}+\delta_{2}$ are bounds of the confidence interval for location parameter $\theta$, and $\tilde{C}(\theta \mid \hat{x})$ is the confidence density.

Such identity (2.7) is a property of statistically self-dual distributions, namely, Cauchy and Cauchy, normal and normal, Laplace and Laplace.

\section{The Transform between the space of observed values and the space of possible values of the parameter}

It is easy to show that the reconstruction of the confidence density is unique if Eqs. (2.3) or (2.7) hold [1], 2].

As a result we have the Transform (both for Poisson-Gamma pair of families of distributions and for statistically self-dual distributions)

$$
\tilde{f}(\theta \mid \hat{x})=T_{c d} \hat{x}
$$

between the space of the realizations $\hat{x}$ of random variable $x$ (with the probability density $f(x \mid \theta)$ ) and the space of the possible values of the parameter $\theta$ (with the confidence density $\tilde{f}(\theta \mid \hat{x})$ ). Here $f(x \mid \theta)$ is the probability density of either normal, or Cachy, or Laplace distribution and $\tilde{f}(\theta \mid \hat{x})$ is the corresponding confidence distribution.

The Transform Eq. (3.1) allows one to use statistical inferences about the random variable for estimation of an unknown parameter.

The simplest examples of this are given by several infinitely divisible distributions.

Definition 2: A distribution $F$ is infinitely divisible if for each $n$ there exist a distribution function $F_{n}$ such that $F$ is the $n$-fold convolution of $F_{n}$.

As it is known the Poisson, Gamma-, normal and Cauchy distributions are infinitely divisible distributions. The sum of independent and identically distributed random variables, which obey one of the families of distributions above, also obeys the distribution from the same family. Application of the Transform Eq. (3.1) to this sum allows one to reconstruct the confidence density of the parameter in the case of several observation of the same random variable. It means that we construct the relation

$$
\tilde{f}\left(n \theta \mid \hat{x}_{1}+\hat{x}_{2}+\ldots+\hat{x}_{n}\right)=T_{c d}\left(\hat{x}_{1}+\hat{x}_{2}+\ldots+\hat{x}_{n}\right),
$$

where $T_{c d}$ is the operator of the Transform Eq. (3.1), the set $\hat{x}_{1}, \hat{x}_{2}, \ldots, \hat{x}_{n}$ are the observed values. Thereafter we reconstruct the confidence density of $\theta$, i.e. $\tilde{f}\left(\theta \mid \hat{x}_{1}, \hat{x}_{2}, \ldots, \hat{x}_{n}\right)$.

The use of the confidence density also can be formulated in Bayesian framework.

Let us consider, as an example, the Cauchy distribution. In our approach we suppose that the parameter $\theta$ is not a random value and before the measurement we do not prefer any of values of 
this parameter, i.e. possible values of the parameter have equal probability and a prior distribution of $\theta$ is $\pi(\theta)=$ const. Suppose we observe $\hat{x}_{1}$ and update our prior via the Transform Eq. (3.1) to obtain $\tilde{C}\left(\theta \mid \hat{x}_{1}\right)$, which is the pdf of the Cauchy distribution. This becomes our new prior before observing $\hat{x}_{2}$. It is easy to show that in the case of the observing $\hat{x}_{2}$ the reconstructed confidence density (or our next new prior) $\tilde{C}\left(2 \theta \mid \hat{x}_{1}+\hat{x}_{2}\right)^{2}$ also is the pdf of the Cauchy distribution. By induction this argument extends to sequences of any number of observations

$$
\tilde{C}\left(n \theta \mid \hat{x}_{1}+\hat{x}_{2}+\ldots+\hat{x}_{n}\right)=T_{c d}\left(\hat{x}_{1}+\hat{x}_{2}+\ldots+\hat{x}_{n}\right),
$$

i.e. we use the iterative procedure

$$
\tilde{C}\left(\theta \mid \hat{x}_{1}, \hat{x}_{2}, \ldots, \hat{x}_{n-1}, \hat{x}_{n}\right)=T_{p d}\left(\tilde{C}\left(\theta \mid \hat{x}_{1}, \hat{x}_{2}, \ldots, \hat{x}_{n-1}\right), \hat{x}_{n}\right),
$$

where $T_{p d}$ is the operator of the Transform between a prior density and a posterior density of the parameter.

Note that a prior density here is only the result of direct calculations of probabilities in frame of the Transform $T_{p d}$ with the usage of the knowledge about the law of distribution of the random variable, i.e. we construct the confidence density without any suppositions about a prior ("uniform prior" is not a prior density because if $\pi(\theta)=$ const then $\left.\int_{-\infty(\text { or } 0)}^{\infty} \pi(\theta) d \theta=\infty\right)$. On the other hand, a prior knowledge about the law of distribution of the parameter in the case of the random origin of parameter can be used for construction of the confidence density.

\section{The method of confi dence density construction for a signal with known background}

The confidence density is more informative notion than the confidence interval and gives many advantages in the construction of the confidence intervals. For example, the Gamma-distribution $\Gamma_{1, \hat{n}+1}$ is the confidence density of the parameter of Poisson distribution in the case of the $\hat{n}$ observed events from the Poisson flow of events [3, 4 . It means that we can reconstruct any confidence intervals (shortest, central, with optimal coverage, ...) by the direct calculation of the probability density of Gamma-distribution.

The next example illustrates the advantages of the confidence density construction. Let us consider the Poisson distribution with two components: the signal component with a parameter $\mu_{s}$ and background component with a parameter $\mu_{b}$, where $\mu_{b}$ is known. To construct confidence intervals for the parameter $\mu_{s}$ of a signal in the case of observed value $\hat{n}$, we must find the confidence density $P\left(\mu_{s} \mid \hat{n}\right)$.

Firstly let us consider the simplest case $\hat{n}=\hat{s}+\hat{b}=1$. Here $\hat{s}$ is the number of signal events and $\hat{b}$ is the number of background events among the observed number $\hat{n}$ of events.

\footnotetext{
${ }^{2}$ As it is known, if $C\left(x_{1} \mid \theta_{1}, b_{1}\right)=\frac{b_{1}}{\pi\left(b_{1}^{2}+\left(x_{1}-\theta_{1}\right)^{2}\right)}$ and $C\left(x_{2} \mid \theta_{2}, b_{2}\right)=\frac{b_{2}}{\pi\left(b_{2}^{2}+\left(x_{2}-\theta_{2}\right)^{2}\right)}$ then $C\left(x_{1}+x_{2} \mid \theta_{1}+\right.$ $\left.\theta_{2}, b_{1}+b_{2}\right)=\frac{b_{1}+b_{2}}{\pi\left(\left(b_{1}+b_{2}\right)^{2}+\left(\left(x_{1}+x_{2}\right)-\left(\theta_{1}+\theta_{2}\right)\right)^{2}\right)}$ with statistically dual distribution $\tilde{C}\left(\theta_{1}+\theta_{2} \mid x_{1}+x_{2}, b_{1}+b_{2}\right)=$ $\frac{b_{1}+b_{2}}{\pi\left(\left(b_{1}+b_{2}\right)^{2}+\left(\left(x_{1}+x_{2}\right)-\left(\theta_{1}+\theta_{2}\right)\right)^{2}\right)}$. It means that we can reconstruct $\tilde{C}\left(\theta \mid \hat{x}_{1}, \hat{x}_{2}\right)$ using $\tilde{C}\left(2 \theta \mid \hat{x}_{1}+\hat{x}_{2}, 2 b\right)$ (in our case $\theta_{1}=\theta_{2}=\theta$ and $\left.b_{1}=b_{2}=b\right)$.
} 
$\hat{b}$ can be equal to 0 and 1 . We know that $\hat{b}$ is equal to 0 with probability (Eq.(2))

$$
p_{0}=f\left(\hat{b}=0 \mid \mu_{b}\right)=\frac{\mu_{b}^{0}}{0 !} e^{-\mu_{b}}=e^{-\mu_{b}}
$$

and $\hat{b}$ is equal to 1 with probability

$$
p_{1}=f\left(\hat{b}=1 \mid \mu_{b}\right)=\frac{\mu_{b}^{1}}{1 !} e^{-\mu_{b}}=\mu_{b} e^{-\mu_{b}}
$$

Correspondingly, $P(\hat{b}=0 \mid \hat{n}=1)=P(\hat{s}=1 \mid \hat{n}=1)=\frac{p_{0}}{p_{0}+p_{1}}$ and

$P(\hat{b}=1 \mid \hat{n}=1)=P(\hat{s}=0 \mid \hat{n}=1)=\frac{p_{1}}{p_{0}+p_{1}}$.

It means that the distribution of the confidence density $P\left(\mu_{s} \mid \hat{n}=1\right)$ is equal to the sum of distributions

$$
P(\hat{s}=1 \mid \hat{n}=1) \Gamma_{1,2}+P(\hat{s}=0 \mid \hat{n}=1) \Gamma_{1,1}=\frac{p_{0}}{p_{0}+p_{1}} \Gamma_{1,2}+\frac{p_{1}}{p_{0}+p_{1}} \Gamma_{1,1},
$$

where $\Gamma_{1,1}$ is the Gamma distribution with the probability density $g_{\hat{s}=0}\left(\mu_{s}\right)=e^{-\mu_{s}}$ and $\Gamma_{1,2}$ is the Gamma distribution with the probability density $g_{\hat{s}=1}\left(\mu_{s}\right)=\mu_{s} e^{-\mu_{s}}$. As a result, we have the confidence density of the parameter $\mu_{s}$

$$
P\left(\mu_{s} \mid \hat{n}=1\right)=\frac{\mu_{s}+\mu_{b}}{1+\mu_{b}} e^{-\mu_{s}}
$$

Using the formula (Eq.(15)) for $P\left(\mu_{s} \mid \hat{n}=1\right)$ and formula (Eq.(4)), we construct the shortest confidence interval of any confidence level in a trivial way.

In this manner we can construct the confidence density $P\left(\mu_{s} \mid \hat{n}\right)$ for any values of $\hat{n}$ and $\mu_{b}$. We have obtained the known formula [13, 14, 15,

$$
P\left(\mu_{s} \mid \hat{n}\right)=\frac{\left(\mu_{s}+\mu_{b}\right)^{\hat{n}}}{\hat{n} ! \sum_{i=0}^{\hat{n}} \frac{\mu_{b}^{i}}{i !}} e^{-\mu_{s}} .
$$

The numerical results for the confidence intervals are shown in Table 1.

\section{The "Inverse Transform"}

In this Section the approach to estimation of quality of planned experiments [9] is used to show the possibility of the "Inverse Transform". This approach is based on the analysis of uncertainty, which will take place under the future hypotheses testing about the existence of a new phenomenon in Nature. We consider a simple statistical hypothesis $H_{0}$ : new physics is present in Nature (i.e. $\mu=$ $\mu_{s}+\mu_{b}$ in the Eq.(2)) against a simple alternative hypothesis $H_{1}$ : new physics is absent $\left(\mu=\mu_{b}\right)$. The value of uncertainty is determined by the values of the probability to reject the hypothesis $H_{0}$ when it is true (Type I error $\alpha$ ) and the probability to accept the hypothesis $H_{0}$ when the hypothesis $H_{1}$ is true (Type II error $\beta$ ). This uncertainty characterises the distinguishability of the hypotheses under the given choice of critical area. 


\begin{tabular}{r|cccccc}
\hline$\hat{n} \backslash \mu_{b}$ & 0.0 & 1.0 & 2.0 & 6.0 & 12.0 & 15.0 \\
\hline 0 & $0.00,2.30$ & $0.00,2.30$ & $0.00,2.30$ & $0.00,2.30$ & $0.00,2.30$ & $0.00,2.30$ \\
1 & $0.09,3.93$ & $0.00,3.27$ & $0.00,3.00$ & $0.00,2.63$ & $0.00,2.48$ & $0.00,2.45$ \\
2 & $0.44,5.48$ & $0.00,4.44$ & $0.00,3.88$ & $0.00,3.01$ & $0.00,2.68$ & $0.00,2.61$ \\
3 & $0.93,6.94$ & $0.00,5.71$ & $0.00,4.93$ & $0.00,3.48$ & $0.00,2.91$ & $0.00,2.78$ \\
4 & $1.51,8.36$ & $0.51,7.29$ & $0.00,6.09$ & $0.00,4.04$ & $0.00,3.16$ & $0.00,2.98$ \\
5 & $2.12,9.71$ & $1.15,8.73$ & $0.20,7.47$ & $0.00,4.71$ & $0.00,3.46$ & $0.00,3.20$ \\
6 & $2.78,11.05$ & $1.79,10.07$ & $0.83,9.01$ & $0.00,5.49$ & $0.00,3.80$ & $0.00,3.46$ \\
7 & $3.47,12.38$ & $2.47,11.38$ & $1.49,10.37$ & $0.00,6.38$ & $0.00,4.19$ & $0.00,3.74$ \\
8 & $4.16,13.65$ & $3.18,12.68$ & $2.20,11.69$ & $0.00,7.35$ & $0.00,4.64$ & $0.00,4.06$ \\
9 & $4.91,14.95$ & $3.91,13.96$ & $2.90,12.94$ & $0.00,8.41$ & $0.00,5.15$ & $0.00,4.42$ \\
10 & $5.64,16.21$ & $4.66,15.22$ & $3.66,14.22$ & $0.02,9.53$ & $0.00,5.73$ & $0.00,4.83$ \\
20 & $13.50,28.33$ & $12.53,27.34$ & $11.53,26.34$ & $7.53,22.34$ & $1.70,16.08$ & $0.00,12.31$
\end{tabular}

Table 1: $90 \%$ C.L. intervals for the Poisson signal mean $\mu_{s}$, for total events observed $\hat{n}$, for known mean background $\mu_{b}$ ranging from 0 to 15 .

Let both values $\mu_{s}$ and $\mu_{b}$, which are defined in the previous Section, be exactly known. In this simplest case the errors of Type I and II, which will take place in testing of hypothesis $H_{0}$ versus hypothesis $H_{1}$, can be written as follows:

$$
\left\{\begin{array}{l}
\alpha=\sum_{i=0}^{n_{c}} f\left(i \mid \mu_{s}+\mu_{b}\right) \\
\beta=1-\sum_{i=0}^{n_{c}} f\left(i \mid \mu_{b}\right)
\end{array}\right.
$$

where $n_{c}$ is a critical value and $f(i \mid \mu)$ is defined by the Eq.(2).

Let the values $\hat{\mu}_{s}=\hat{s}$ and $\hat{\mu}_{b}=\hat{b}$ be known, for example, from Monte Carlo experiment with integral luminosity which is exactly the same as the data luminosity later in the planned experiment. It means that we must include the uncertainties in values $\mu_{s}$ and $\mu_{b}$ to the system of the equations Eqs.(17). As it is shown in ref. [9] (see, also, the generalised case in the same reference) we have the system

$$
\left\{\begin{array}{l}
\alpha=\int_{0}^{\infty} g_{\hat{s}+\hat{b}}(\lambda) \sum_{i=0}^{n_{c}} f(i \mid \lambda) d \lambda=\sum_{i=0}^{n_{c}} \frac{C_{\hat{s}+\hat{b}+i}^{i}}{2^{\hat{s}+\hat{b}+i+1}} \\
\beta=1-\int_{0}^{\infty} g_{\hat{b}}(\lambda) \sum_{i=0}^{n_{c}} f(i \mid \lambda) d \lambda=1-\sum_{i=0}^{n_{c}} \frac{C_{\hat{b}+i}^{i}}{2^{\hat{b}+i+1}}
\end{array}\right.
$$

where the critical value $n_{c}$ under the future hypotheses testing about the observability can be chosen in accordance with the test of equal probability [16] and $C_{N}^{i}$ is $\frac{N !}{i !(N-i) !}$.

Note, here the Poisson distribution is a prior distribution of the expected probabilities and the negative binomial (Pascal) distribution is a posterior distribution of the expected probabilities of the random variable. This transformation of the estimated confidence densities $g_{\hat{s}+\hat{b}}(\lambda)$ and $g_{\hat{b}}(\lambda)$ 
(probability densities of the corresponding $\Gamma$-distributions) to the space of the expected values of the random variable can be named the "Inverse Transform".

\section{Conclusions}

We have shown that the statistical duality allows one to connect the estimation of the parameter with the measurement of the random variable of the distribution due to the Transform Eq. (3.1). It gives the tool to construct the confidence densities.

The usage of the confidence densities for the construction of the confidence intervals and for the construction of a posterior distributions of probabilities is presented in examples.

\section{Acknowledgments}

The authors are grateful to V.B. Gavrilov, V.A. Kachanov, Louis Lyons, V.A. Matveev and V.F. Obraztsov for the interest and useful comments, Yu.P. Gouz, A. Nikitenko and C. Wulz for fruitful discussions.

\section{References}

[1] S.I. Bityukov, V.A. Taperechkina, V.V. Smirnova, Statistically dual distributions and estimation of the parameters math.ST/0411462, 2004.

[2] S.I. Bityukov, N.V. Krasnikov, Statistically dual distributions and conjugate families, in Bayesian Inference and Maximum Entropy Methods in Science and Engineering, edited by K. H. Knuth, A. E. Abbas, R. D. Morris, J. P. Castle, AIP Conference Proceedings 803 (2005) 398, Melville, NY.

[3] S.I. Bityukov, N.V. Krasnikov, V.A. Taperechkina, Confidence intervals for Poisson distribution parameter, Preprint IFVE 2000-61, Protvino, 2000 [hep-ex/0108020].

[4] S.I.Bityukov, On the Signal Significance in the Presence of Systematic and Statistical Uncertainties, JHEP 09 (2002) 060 [hep-ph / 0207130 ]; S.I. Bityukov, N.V. Krasnikov, Signal Significance in the Presence of Systematic and Statistical Uncertainties Nucl.Inst.\&Meth. A502, (2003) 795.

[5] B. Efron, R.A. Fisher in the 21st Century, Stat.Sci. 13 (1998) 95.

[6] R.A. Fisher, Inverse probability, in Proc. of the Cambridge Philosophical Society 26 (1930) 528.

[7] K. Singh, M. Xie, W.E. Strawderman, Combining information from independent sources through confidence distributions, The Annals of Statistics 33 (2005) 159.

[8] S.I. Bityukov, N.V. Krasnikov, V.A. Taperechkina, V.V. Smirnova, Statistically dual distributions in statistical inference, in proceedings of Statistical problems in Particle Physics, Astrophysics and Cosmology, September 2005, Oxford, UK, Imperial College Press, 2006, p.102.

[9] S.I. Bityukov, N.V. Krasnikov, The probability of making a correct decision in hypotheses testing as estimator of quality of planned experiments, in Bayesian Inference and Maximum Entropy Methods in Science and Engineering, edited by G. Erickson, Y. Zhai, AIP Conference Proceedings 707 (2004) 455, Melville, NY.

[10] E.T. Jaynes: Papers on probability, statistics and statistical physics, Ed. by R.D. Rosenkrantz, D.Reidel Publishing Company, Dordrecht, Holland, p.165, 1983. 
[11] A.G.Frodesen, O.Skjeggestad, H.Toft, Probability and Statistics in Particle Physics, UNIVERSITETSFORLAGET, Bergen-Oslo-Tromso, p.97, 1979.

[12] R.D. Cousins, Why isn't every physicist a Bayesian? Am.J.Phys., 63 (1995) 398.

[13] G.P. Yost et.al., Rev.Part.Prop., Phys.Lett. B204 (1988) p.81 (formula O. Helene).

[14] G. Zech, Upper Limits in Experiments with Background or Measurement Errors, Nucl.Inst.\&Meth. A 277 (1989) 608..

[15] G. D’Agostini, Bayesian Reasoning in High-Energy Physics: Principles and Applications, Yellow Report CERN 99-03, Geneva, Switzerland, p.95 (1999) [hep-ph/9512295, p.70].

[16] S.I. Bityukov, N.V. Krasnikov, On Observability of Signal above Background, Nucl.Instr.\&Meth. A452 (2000) 518. 